\title{
Assessing Real World Effect of Laparoscopic Bariatric Surgery on Healthcare Costs- a Retrospective Matched Cohort Study using a U.S. Administrative Claims Database
}

\author{
${ }^{1}$ Willis Towers Watson, Milwaukee, WI \\ ${ }^{2}$ Greenville Health System, Greenville, SC \\ 3Johnson \& Johnson, New Brunswick, NJ \\ ${ }^{4}$ Ethicon, Somerville, NJ
}

John Dawson ${ }^{1}$, Eric Bour², Andrew $\mathrm{Yoo}^{3}$, Gang Li ${ }^{3}$, Natalie Heidrich ${ }^{4}$ and Anuprita Patkar ${ }^{4 *}$

Received: November 05, 2016; Accepted: November 15, 2016; Published: December 08, 2016

*Corresponding author: Anuprita D. Patkar, PhD, Director, Global Health Economics \& Market Access, Ethicon Surgical Care, Johnson \& Johnson, Route 22 West, Somerville, NJ 08876,Tel:908-218-5625;Email: apatkar2@its.jnj.com

\begin{abstract}
Background: Bariatric surgery is considered an efficacious procedure for stimulating significant weight loss and improving obesity-related co morbidities among severely obese persons; however, the effect of bariatric surgery on healthcare costs is debated.

Objective: To evaluate the effect of 3 types of laparoscopic bariatric surgery procedures on healthcare costs.

Methods: Patient's $\geq 18$ years of age who had laparoscopic bariatric surgery between January 2006 and December 2013were identified from the optum clinformatics administrative claims database. Patients who were identified as having had Laparoscopic Adjustable Gastric Banding (LAGB), Gastric Bypass (LRYGB), or Sleeve Gastrectomy (LSG), were matched to medically-managed patients (controls) based on age, sex, obesity category, insurance type, obesity-related co morbidities, and baseline average monthly healthcare cost. Medication costs for treatment of selected obesityrelated co morbidities (type 2 diabetes [T2DM], dyslipidemia, and hypertension), (non-medication) medical costs, and the combination of these costs (combined treatment costs) were measured for every 6 months up to 5 years after the surgery or an assigned index date for controls (followup). Healthcare costs were compared between the surgical and control cohorts using mixed models for repeated measurements.

Results: Patients who had LAGB ( $N=4,208$, mean age $=46.3$ years), LRYGB $(\mathrm{N}=4,308$, mean age $=46.4$ years $)$, and LSG ( $N=545$, mean age=45.1 years) and patients in the control cohort ( $\mathrm{N}=9,061$, mean age $=46.4$ years $)$ were similar in age and the majority of patients in each study cohort were female (69.4\%-75.8\%).By the $5^{\text {th }}$ year of follow-up, all categories of adjusted monthly healthcare costs were significantly lower for the LRYGB cohort than the control cohort (medication, $\$ 156$ vs. $\$ 245, \mathrm{P}<0.001$; medical, $\$ 650$ vs. $\$ 1,019, \mathrm{P}<0.001$; combined treatment, $\$ 977$ for LRYGB vs. $\$ 1,354$ for controls, $P=0.001$ ). Likewise, adjusted monthly healthcare costs were significantly lower for the LSG cohort than the control, but the LSG results are inclusively because of the recent uptake of LSG. The adjusted monthly combined treatment and medical costs of the LAGB cohort tended to not differ significantly from those of the control cohort; however, by the end of follow- this cohort had significantly lower medication costs (\$208 for LAGB vs. $\$ 245$ for controls, $\mathrm{P}=0.030$ ). The magnitude and statistical significance of cost differences in sub-analyses of patients with selected obesity-related co morbidities tended to be greater and tended to indicate lower costs in the surgical cohorts by the end of follow-up. Time to full ROI was realized for the LRYGB cohort in approximately 4 years among patients with T2DM and in approximately 3 years among patients with T2DM using insulin.

Conclusion: In this retrospective analysis of a large real-world claims database, patients who had laparoscopic bariatric surgery incurred lower medication costs for the treatment of T2DM, dyslipidemia, and hypertension compared to medically-managed controls up to 5 years post-surgery. LRYGB was associated with the greatest reduction in medication costs and sustained lower combined treatment costs in comparison to medicallymanaged controls over the 5 year follow-up period. LAGB did not demonstrate sustained lower cost vs control, and more study is needed to evaluate the long term impact of LSG.
\end{abstract}

\section{Introduction}

According to the data from the National Health and Nutrition Examination Survey (2009-2010), 35.7\% of adults in the U.S. were obese (body mass index (BMI): $\geq 30 \mathrm{~kg} / \mathrm{m}^{2}$ ) and $6.3 \%$ had severe obesity (BMI: $\geq 40 \mathrm{~kg} / \mathrm{m}^{2}$ ).[1]The association between severe obesity and adverse health risks has been well documented.[2] The guidelines of the American Society for Metabolic and Bariatric Surgery(ASMBS) report that severe obesity may be associated with Type 2 Diabetes (T2DM), dyslipidemia, hypertension, stroke, heart disease, cancer, asthma, coronary artery disease, 
gallstones, gastro esophageal reflux, hypertension, nonalcoholic steatohepatitis or nonalcoholic fatty liver disease, sleep apnea, urinary incontinence, breast cancer, congestive heart failure, lymphedema, major depression, osteoarthritis, polycystic ovary syndrome, pseudo tumorcerebri, and venous stasis or leg ulcers. ${ }^{2}$ In the U.S., the medical costs associated with obesity were estimated at $\$ 209.7$ billion annually in 2008 dollars, with the majority of the costs attributed to treating cardiovascular disease and T2DM.[3-5]

Bariatric surgery is considered an effective procedure for stimulating significant weight loss and improving obesityrelated co morbidities such as T2DM among severely obese persons.[2,6]However, in 2010 only 1\% of persons clinically eligible for bariatric surgery received surgical treatment.[7] Using the Nationwide Inpatient Sample database, the number of bariatric surgeries performed in the U.S. from 2008 to 2012 was estimated at 598,576, with a rate in 2012 of 51 surgeries per 100,000 people.[8]Current guidelines of the American Association of Clinical Endocrinologists, The Obesity Society, and the ASMBS recommend that persons with severe obesity $(\geq 40$ $\mathrm{kg} / \mathrm{m}^{2}$ ) without coexisting medical problems and persons with a BMI $\geq 35 \mathrm{~kg} / \mathrm{m}^{2}$ and at least one obesity related co morbidity (e.g. T2DM, dyslipidemia, hypertension) be considered eligible for bariatric surgery.[6]A Cochrane review of 22 randomized controlled trials with 1,798 participants reported that bariatric surgery was associated with greater improvement in weight loss outcomes and weight-associated co morbidities when compared with non-surgical interventions, regardless of the type of procedure used.[9]Bariatric surgery techniques have evolved over the last decade and the majority are performed using the laparoscopic approach, which is associated with shorter hospital stays and lower complication rates than open gastric bypass. $[8,10,11]$

Results of a large observational study, conducted by Weiner et al, of 29,820 plan members who underwent bariatric surgery between 2002 and 2008 indicated that bariatric surgery is associated with a reduction in overall healthcare costs in the first years following surgery; however, the savings in healthcare costs of patients who underwent surgery versus those of non-surgery controls did not persist.[12]Although costs for pharmacy and outpatient visits did remain reduced among surgery patients versus controls up to 6 years post-surgery in that study, inpatient services were higher and offset the savings.[12] The findings from Weiner et al., which included a significant number of open gastric bypass cases with more and longer inpatient care, are becoming less pertinent in more recent years where the increasing majority of bariatric surgeries are performed laparoscopically. [12] The present study aimed to specifically evaluate the effect of 3 types of laparoscopic bariatric surgery procedures-Adjustable Gastric Banding (LAGB), Gastric Bypass (LRYGB), and Sleeve Gastrectomy (LSG)—on healthcare costs.

\section{Methods}

\section{Data Source}

This study was based on administrative insurance claims data for the time period spanning January 1, 2005 through December
31, 2013, drawn from the optum clinformatics database. The optum clinformatics database comprises enrollment information, inpatient medical, outpatient medical, and outpatient prescription claims data for tens of millions of individuals in the U.S. annually. As described in greater detail below, International Classification of Diseases, 9th revision, Clinical Modification (ICD-9-CM) codes, Current Procedural Terminology (CPT) codes, and National Drug Codes (ndcs) were used to identify the study population and measure healthcare cost outcomes.

\section{Study Population \&Design \\ Identification of Laparoscopic Bariatric Surgeries}

The cohorts of patients who had laparoscopic bariatric surgery procedures (henceforth, surgical cohorts)included individuals $\geq 18$ years of age who had LAGB, LRYGB, or LSG between January 1, 2006 and December 31, 2013, identified through insurance claims with ICD-9-CM and CPT procedure codes for the surgeries.

\section{Study Periods}

Definitions of the various study periods are provided in Supplemental Table 1 and described below. For each surgical cohort patient, the date of the first observed laparoscopic bariatric surgery was designated the index date. Patients were assigned to each surgical cohort based on the type of surgery (index surgery) that they had on the index date: LAGB, LRYGB, or LSG. Patients were required to have continuous enrollment in their insurance throughout the following periods: [1] a 360-day baseline period immediately before the index date, [2] a 60-day post-surgery recovery period which commenced upon the index date (established to allow the bariatric surgery cohort to stabilize prior to beginning measurement of outcomes), and [3] for at least 360 days after the termination of the 60-day post-surgery recovery period; this latter period was designated as the followup period which lasted for as long as each patient remained in the insurance plan, up to 5 years. The 5 -year follow-up period was sub-divided into 10 half-yearly panels such that a patient enrolled in their insurance for 2 years after the post-surgery recovery period would contribute 4 half-yearly panels, whereas a patient enrolled for 5 years would contribute 10 half-yearly panels. The follow-up period was shorter for the LSG cohort, between 2-4 years depending on the outcome measure, due to a recent uptake of the procedure.

\section{Eligibility Criteria for Surgical Cohorts}

The following additional inclusion criteria were applied to patients in the surgical cohorts: [1] $\geq 1$ medical claim with an ICD-9-CM diagnosis code for obesity or severe obesity during the baseline period; [2] evidence of $\geq 1$ obesity-related co morbidity (type 2 diabetes, dyslipidemia, hypertension, depression, or sleep apnea), as indicated by having either at least two separate medical claims with an ICD-9-CM diagnosis code for the co morbidity, or one such medical claim plus at $\geq 1$ pharmacy claim for a medication used to manage the co morbidity, during the baseline period.

The following exclusion criteria were applied to patients in the surgical cohorts: [1] $\geq 1$ medical claim indicative of pregnancy 
Assessing Real World Effect of Laparoscopic Bariatric Surgery on Healthcare Costs- a Retrospective Matched Cohort Study using a U.S. Administrative Claims Database

Table 1: Baseline demographics and clinical characteristics, all patients in surgical and control cohorts

\begin{tabular}{|c|c|c|c|c|c|c|c|c|c|c|c|c|c|c|}
\hline \multirow{2}{*}{ Age (years) } & \multicolumn{2}{|c|}{$\begin{array}{c}\text { LAGB } \\
\mathrm{N}=4,208\end{array}$} & \multicolumn{2}{|c|}{$\begin{array}{c}\text { LAGB } \\
\text { Control } \\
\mathrm{N}=4,208\end{array}$} & \multicolumn{2}{|c|}{$\begin{array}{c}\text { LRYGB } \\
\mathrm{N}=4,308\end{array}$} & \multicolumn{2}{|c|}{$\begin{array}{c}\text { LRYGB } \\
\text { Control } \\
\mathbf{N}=4,308\end{array}$} & \multicolumn{2}{|c|}{$\begin{array}{c}\text { LSG } \\
N=545\end{array}$} & \multicolumn{2}{|c|}{$\begin{array}{c}\text { LSG } \\
\text { Control } \\
\mathrm{N}=545\end{array}$} & \multicolumn{2}{|c|}{$\begin{array}{c}\text { Combined } \\
\text { Control } \\
\mathrm{N}=9,061\end{array}$} \\
\hline & & & & & & & & & & & & & & \\
\hline Mean (Stdev) & \multicolumn{2}{|c|}{$46.3(9.9)$} & \multicolumn{2}{|c|}{$46.4(9.8)$} & \multicolumn{2}{|c|}{$46.4(9.8)$} & \multicolumn{2}{|c|}{$46.5(9.7)$} & \multicolumn{2}{|c|}{$45.1(9.7)$} & \multicolumn{2}{|c|}{$45.1(9.6)$} & \multicolumn{2}{|c|}{$46.4(9.8)$} \\
\hline Median & \multicolumn{2}{|c|}{47} & \multicolumn{2}{|c|}{47} & \multicolumn{2}{|c|}{47} & \multicolumn{2}{|c|}{47} & \multicolumn{2}{|c|}{45} & \multicolumn{2}{|c|}{46} & \multicolumn{2}{|c|}{47} \\
\hline \multicolumn{15}{|l|}{$\begin{array}{l}\text { Age group } \\
\text { (years) }\end{array}$} \\
\hline$\leq 30$ & 260 & $6.20 \%$ & 241 & $5.70 \%$ & 221 & $5.10 \%$ & 215 & $5.00 \%$ & 35 & $6.40 \%$ & 38 & $7.00 \%$ & 494 & $5.50 \%$ \\
\hline $31-40$ & 1,003 & $23.80 \%$ & 1,003 & $23.80 \%$ & 1,070 & $24.80 \%$ & 1,026 & $23.80 \%$ & 142 & $26.10 \%$ & 137 & $25.10 \%$ & 2,166 & $23.90 \%$ \\
\hline $41-50$ & 1,417 & $33.70 \%$ & 1,429 & $34.00 \%$ & 1,432 & $33.20 \%$ & 1,462 & $33.90 \%$ & 198 & $36.30 \%$ & 202 & $37.10 \%$ & 3,093 & $34.10 \%$ \\
\hline $51-60$ & 1,247 & $29.60 \%$ & 1,248 & $29.70 \%$ & 1,291 & $30.00 \%$ & 1,321 & $30.70 \%$ & 142 & $26.10 \%$ & 140 & $25.70 \%$ & 2,709 & $29.90 \%$ \\
\hline$\geq 61$ & 281 & $6.70 \%$ & 287 & $6.80 \%$ & 294 & $6.80 \%$ & 284 & $6.60 \%$ & 28 & $5.10 \%$ & 28 & $5.10 \%$ & 599 & $6.60 \%$ \\
\hline Female & 3,139 & $74.60 \%$ & 3,139 & $74.60 \%$ & 3,265 & $75.80 \%$ & 3,265 & $75.80 \%$ & 378 & $69.40 \%$ & 378 & $69.40 \%$ & 6,782 & $74.80 \%$ \\
\hline \multicolumn{15}{|l|}{ Insurance type } \\
\hline Fee for Service & 3,820 & $90.80 \%$ & 3,820 & $90.80 \%$ & 3,602 & $83.60 \%$ & 3,602 & $83.60 \%$ & 521 & $95.60 \%$ & 521 & $95.60 \%$ & 7,943 & $87.70 \%$ \\
\hline HMO & 386 & $9.20 \%$ & 386 & $9.20 \%$ & 706 & $16.40 \%$ & 706 & $16.40 \%$ & 24 & $4.40 \%$ & 24 & $4.40 \%$ & 1,116 & $12.30 \%$ \\
\hline Other/unknown & 2 & $0.00 \%$ & 2 & $0.00 \%$ & 0 & $0.00 \%$ & 0 & $0.00 \%$ & 0 & $0.00 \%$ & 0 & $0.00 \%$ & 2 & $0.00 \%$ \\
\hline \multicolumn{15}{|l|}{ BMI } \\
\hline $30-40 \mathrm{~kg} / \mathrm{m} 2$ & 505 & $12.00 \%$ & 505 & $12.00 \%$ & 493 & $11.40 \%$ & 493 & $11.40 \%$ & 61 & $11.20 \%$ & 61 & $11.20 \%$ & 1,059 & $11.70 \%$ \\
\hline$\geq 40 \mathrm{~kg} / \mathrm{m} 2$ & 3,703 & $88.00 \%$ & 3,703 & $88.00 \%$ & 3,815 & $88.60 \%$ & 3,815 & $88.60 \%$ & 484 & $88.80 \%$ & 484 & $88.80 \%$ & 8,002 & $88.30 \%$ \\
\hline \multicolumn{15}{|l|}{$\begin{array}{l}\text { Obesity-related } \\
\text { comorbidities }\end{array}$} \\
\hline Type 2 Diabetes & 1,321 & $31.40 \%$ & 1,321 & $31.40 \%$ & 1,664 & $38.60 \%$ & 1,664 & $38.60 \%$ & 172 & $31.60 \%$ & 172 & $31.60 \%$ & 3,157 & $34.80 \%$ \\
\hline Dyslipidemia & 2,157 & $51.30 \%$ & 2,157 & $51.30 \%$ & 2,241 & $52.00 \%$ & 2,241 & $52.00 \%$ & 262 & $48.10 \%$ & 262 & $48.10 \%$ & 4,660 & $51.40 \%$ \\
\hline Hypertension & 3,006 & $71.40 \%$ & 3,006 & $71.40 \%$ & 3,152 & $73.20 \%$ & 3,152 & $73.20 \%$ & 397 & $72.80 \%$ & 397 & $72.80 \%$ & 6,555 & $72.30 \%$ \\
\hline Depression & 1,145 & $27.20 \%$ & 1,145 & $27.20 \%$ & 1,383 & $32.10 \%$ & 1,383 & $32.10 \%$ & 138 & $25.30 \%$ & 138 & $25.30 \%$ & 2,664 & $29.40 \%$ \\
\hline Sleep Apnea & 1,654 & $39.30 \%$ & 1,654 & $39.30 \%$ & 2,055 & $47.70 \%$ & 2,055 & $47.70 \%$ & 248 & $45.50 \%$ & 248 & $45.50 \%$ & 3,951 & $43.60 \%$ \\
\hline \multicolumn{15}{|l|}{ Monthly cost** } \\
\hline Mean (Stdev) & \multicolumn{2}{|c|}{$\$ 925(\$ 1,189)$} & $\$ 925($ & $1,188)$ & $\$ 995$ & $\$ 1,151)$ & $\$ 995$ & $\$ 1,150)$ & $\$ 906$ & $(\$ 1,050)$ & $\$ 905$ & $(\$ 1,078)$ & $\$ 957$ & $\$ 1,164)$ \\
\hline Median & & 54 & & & & 51 & & 652 & & 592 & & 595 & & 602 \\
\hline
\end{tabular}

BMI, body mass index; HMO, health maintenance organization; LAGB, laparoscopic adjustable gastric band; LRYGB, laparoscopic Roux-en-Y gastric bypass; LSG, laparoscopic sleeve gastrectomy; Stdev, standard deviation;

*Values reported are $\mathrm{N}, \%$ unless otherwise noted in rows

** Monthly total healthcare costs measured during the baseline period, excluding the 90 days immediately prior to the index date, which may be a period of intense resource utilization for the surgical cohort patients

or delivery within the baseline, post-surgery recovery, or followup periods; [2] underwent any other bariatric surgery, including open, unknown, or revision surgery during the baseline period or the same encounter in which the index surgery was performed; [3] underwent any other major gastrointestinal surgery during same encounter in which the index surgery was performed; [4] underwent gastric procedures or had medical claims with ICD-9-CM diagnosis codes which were indicative of cancer or benign neoplasms of esophagus, stomach, pancreas, or small intestine that are contraindications for or interfere with bariatric procedures during the baseline period.

\section{Control Cohort}

A control cohort, comprising medically-managed patients without bariatric surgery of any kind, was established for comparison to the surgical cohorts. For each patient in each surgical cohort, potential controls were initially identified by selecting patients who were enrolled in their insurance on the same day that the surgical cohort patient underwent their index surgery, as well as for the equivalent timeframe of the baseline, post-surgical recovery, and minimum 360-day follow-up periods; the total duration of the follow-up period was not required to be exactly the same between the surgical cohort patients and their potential controls. Where applicable, potential control cohort patients were required to meet the same eligibility criteria as the surgical cohort patients (e.g., no cancer).

Matching of potential surgical cohort patients to control cohort patients was performed, at a 1:1 matching ratio, on sex, baseline BMI category (obesity or severe obesity), baseline co morbidities (type 2 diabetes, dyslipidemia, hypertension, depression, sleep apnea), and insurance type (health maintenance organization, fee-for-service, or unknown). Preferential matching of control patients to surgical patients was then performed based on average monthly healthcare costs (within the lower of $15 \%$ or $\$ 150$ ) measured during the baseline period (excluding the 90 days immediately prior to the index date, which may be a period 
of intense resource utilization for the surgical cohort patients) and age (within 5 years).

\section{Evaluation of Healthcare Costs}

For each patient in the study cohorts, repeated measures of healthcare costs were created for the baseline and halfyearly panels in the follow-up period. As noted above, baseline healthcare costs measurements excluded the 90 days immediately prior to the index date, which may be a period of intense resource utilization for the surgical cohort patients. In each period, total (non-medication) medical costs (i.e., costs for all medical claims) and costs for medications specifically used for the treatment of T2DM, dyslipidemia, and hypertension were measured. Medication costs were limited to the medications specifically used for the treatment of those three conditions only because the progression of those conditions is likely altered by bariatric surgery. On the other hand, all medical costs were included in the analysis because the attribution of individual medical services to specific conditions can rarely be achieved reliably in insurance claims due to potential for coding error. A third category, henceforth referred to as 'combined treatment costs,' was equal to the sum of the two categories described above (medication costs plus medical costs). All costs were inflation adjusted to 2013 U.S. dollars.

\section{Statistical Analyses}

Descriptive statistics were utilized to describe the baseline demographics and clinical characteristics of the study cohorts. The control cohorts were similar with respect to baseline characteristics and were pooled together for subsequent analyses. Mixed Models For Repeated Measures (MMRM) were used to compare the healthcare cost outcomes between the surgical and control cohorts. These models included, as independent variables, fixed effects for the patient cohorts (treating the control cohort as reference), sequential panel, a cohort-by-sequential panel interaction, the baseline Charlson Co morbidity Index, and the baseline count of medications used for T2DM, dyslipidemia, and hypertension. An unstructured covariance was used to model the within-patient errors. The Least Squares Means (LSM) method was used to generate adjusted values of each healthcare cost outcome at each time period from the MMRM model results. Analyses were conducted among all patients, and in the following sub-groups of interest: [1] patients with T2DM, dyslipidemia, and hypertension; [2] patients with T2DM; and [3] patients with T2DM using insulin.

\section{Results}

\section{Baseline Demographics and Clinical Characteristics}

Baseline demographics and clinical characteristics of the 6 matched study cohorts and combined control cohort are presented in Table 1. Patients who underwentlagb $(\mathrm{N}=4,208$, mean age: 46.3 years), LRYGB $(\mathrm{N}=4,308$, mean age: 46.4 years), and LSG ( $N=545$, mean age 45.1 years) and patients in the combined control cohort ( $N=9,061$, mean age 46.4 years) were similar in age and the majority of patients in each study cohort were female (69.4\%-75.8\%). Approximately $88 \%$ of patients in each study cohort had a baseline BMI $>40 \mathrm{~kg} / \mathrm{m}^{2}$. In the surgical cohorts, the prevalence of T2DM ranged from $31.4 \%$ to $38.6 \%$, dyslipidemia ranged from $48.1 \%$ to $52.0 \%$, and hypertension ranged from $71.4 \%$ to $73.2 \%$. In the control cohort, the prevalence of T2DM, dyslipidemia, and hypertension, were $34.8 \%, 51.4 \%$, and $72.3 \%$, respectively.

\section{Healthcare Costs among All Patients}

The results of the adjusted analyses of healthcare costs among all patients in the study cohorts are shown in Table 2. Adjusted monthly medical costs and adjusted monthly combined treatment costs of the LAGB, LSG, and control cohorts generally increased over the follow-up period. In the LRYGB cohort, adjusted monthly combined treatment costs decreased from $\$ 995$ during the baseline period to a low of $\$ 899$ at 2.5 years post index, but then gradually increased. At 5 years of follow-up, the adjusted monthly combined treatment costs of the lrygb cohort were significantly lower than those of the control cohort $\$ \$ 977$ vs. $\$ 1,354, P=0.001]$. Also in the LRYGB cohort, the adjusted monthly medical costs increased from the baseline period [\$693], but then began to decrease such that at 5 years of follow-up they were $\$ 650$ and significantly lower than those of controls $[\$ 1,019$, $P<0.01]$. Adjusted monthly medical costs gradually increased over follow-up for the LAGB and control cohorts. Although adjusted monthly medication costs for T2DM, dyslipidemia, and hypertension were higher in the LAGB and lrygbcohorts than the control cohort during the baseline period, they were significantly lower than those of the control cohort at 5 years of follow-up [LAGB: $\$ 208$ [ $P=0.03]$; LRYGB: $\$ 156[P<0.001]$; control: $\$ 245]$. Adjusted monthly medication costs were also significantly lower for the lsgcohort than the control cohort at 2.5 years of followup; however, due to few observations and short follow-up period it was not possible to draw meaningful results for this study population beyond 2.5 years.

\section{Healthcare Costs among Patients with T2DM, Dyslipidemia, and hypertension}

The results of the adjusted analyses of healthcare costs among patients with T2DM, dyslipidemia, and hypertension are shown in Table 3.In the LRYGB cohort, adjusted monthly combined treatment costs decreased from $\$ 1,517$ during the baseline period to $\$ 1,060$ at one year of follow-up, but then gradually increased to $\$ 1,527$ at 5 years of follow-up. At 5 years of follow-up the adjusted monthly combined treatment costs were significantly lower for both the LAGB cohort $[\$ 1,597, P<0.01]$ and the lrygbcohort $[\$ 1,527, P<0.01]$ than controls $[\$ 2,315]$. Adjusted monthly medical costs increased over time in all study cohorts. Although during the baseline period the adjusted monthly medication costs for T2DM, dyslipidemia, and hypertension were higher in the LAGB and lrygbcohorts than the control cohort, they were significantly lower than the control cohort during followup. Patients who underwent LRYGB had the greatest reductions in adjusted monthly medication costs. The savings in medication costs were greater in the sub-group of patients with T2DM, 
Table 2: Adjusted analyses of healthcare costs (constant 2013 USD) among all patients Half-yearly panel period after end of 60-day post-surgery recovery period

\begin{tabular}{|c|c|c|c|c|c|c|c|c|c|c|c|}
\hline cohort & $\begin{array}{l}\text { Index minus } \\
\text { 180days }\end{array}$ & $0-0.5 y$ & $>0.5 y-1 y$ & $>1 y-1.5 y$ & $>1.5-2 y$ & $>2 y-2.5 y$ & $>2.5 y-3 y$ & $>3 y-3.5 y$ & $>3.5 y-4 y$ & $>4 y-4.5 y$ & $>4.5 y-5 y$ \\
\hline \multicolumn{12}{|c|}{ Adjusted Monthly Combined Treatment Costs*, LSM** } \\
\hline LAGB & $\$ 925$ & $\$ 934$ & $\$ 979$ & $\$ 1,084$ & $\$ 1,056$ & $\$ 1,103$ & $\$ 1,176$ & $\$ 1,197$ & $\$ 1,256$ & $\$ 1,460$ & $\$ 1,174$ \\
\hline LRYGB & $\$ 995$ & $\$ 957$ & $\$ 956$ & $\$ 964$ & $\$ 948$ & $\$ 899$ & $\$ 974$ & $\$ 954$ & $\$ 1,016$ & $\$ 1,107$ & $\$ 977$ \\
\hline LSG & $\$ 906$ & $\$ 866$ & $\$ 950$ & $\$ 865$ & $\$ 1,021$ & $\$ 856$ & - & - & - & - & - \\
\hline Control & $\$ 957$ & $\$ 1,010$ & $\$ 972$ & $\$ 1,023$ & $\$ 1,061$ & $\$ 1,056$ & $\$ 1,068$ & $\$ 1,144$ & $\$ 1,171$ & $\$ 1,219$ & $\$ 1,354$ \\
\hline \multicolumn{12}{|c|}{ P-values vs. control cohort } \\
\hline LAGB & $14.00 \%$ & 0 & $76.00 \%$ & 0 & $91.00 \%$ & 0.4 & $9.00 \%$ & 0.51 & $24.00 \%$ & 0 & $17.00 \%$ \\
\hline LRYGB & $8.00 \%$ & 0 & $99.00 \%$ & 0 & $<0.01$ & $<0.001$ & $4.00 \%$ & $<0.01$ & $2.00 \%$ & 0 & $0.10 \%$ \\
\hline LSG & $32.00 \%$ & 0 & $87.00 \%$ & 0 & $72.00 \%$ & 0.21 & - & - & - & - & - \\
\hline \multicolumn{12}{|c|}{ Adjusted Monthly Medical Costs*, LSM** } \\
\hline LAGB & $\$ 658$ & 732 & $\$ 780$ & $\$ 868$ & $\$ 839$ & $\$ 870$ & $\$ 928$ & $\$ 951$ & $\$ 986$ & $\$ 1,173$ & $\$ 880$ \\
\hline LRYGB & $\$ 693$ & $\$ 818$ & $\$ 828$ & $\$ 824$ & $\$ 768$ & $\$ 684$ & $\$ 739$ & $\$ 688$ & $\$ 729$ & $\$ 783$ & $\$ 650$ \\
\hline LSG & $\$ 679$ & $\$ 725$ & $\$ 814$ & $\$ 700$ & $\$ 848$ & $\$ 670$ & - & - & - & - & - \\
\hline Control & $\$ 735$ & $\$ 763$ & $\$ 723$ & $\$ 772$ & $\$ 802$ & $\$ 779$ & $\$ 777$ & $\$ 844$ & $\$ 844$ & $\$ 893$ & $\$ 1,019$ \\
\hline \multicolumn{12}{|c|}{ P-values vs. control cohort } \\
\hline LAGB & $<0.001$ & 0.5 & 0.18 & 0.04 & 0.44 & 0.08 & 0.01 & 0.18 & 0.07 & 0.02 & 0.3 \\
\hline LRYGB & $3.00 \%$ & 0.23 & $1.00 \%$ & 0.27 & $49.00 \%$ & 0.08 & $54.00 \%$ & 0.06 & $17.00 \%$ & 0 & $<0.01$ \\
\hline LSG & $21.00 \%$ & 1 & $37.00 \%$ & 1 & $71.00 \%$ & 0.46 & - & - & - & - & - \\
\hline \multicolumn{12}{|c|}{ Adjusted Monthly Medication Costs*, LSM** } \\
\hline LAGB & $\$ 267$ & $\$ 210$ & $\$ 207$ & $\$ 208$ & $\$ 202$ & $\$ 199$ & $\$ 207$ & $\$ 205$ & $\$ 217$ & $\$ 213$ & $\$ 208$ \\
\hline LRYGB & $\$ 302$ & $\$ 155$ & $\$ 145$ & $\$ 151$ & $\$ 154$ & $\$ 153$ & $\$ 156$ & $\$ 159$ & $\$ 169$ & $\$ 173$ & $\$ 156$ \\
\hline LSG & $\$ 228$ & $\$ 147$ & $\$ 143$ & $\$ 144$ & $\$ 163$ & $\$ 165$ & - & - & - & - & - \\
\hline Control & $\$ 222$ & $\$ 249$ & $\$ 249$ & $\$ 254$ & $\$ 252$ & $\$ 250$ & $\$ 249$ & $\$ 251$ & $\$ 251$ & $\$ 246$ & $\$ 245$ \\
\hline \multicolumn{12}{|c|}{ P-values vs. control cohort } \\
\hline LAGB & $<0.001$ & $<0.001$ & $<0.001$ & $<0.001$ & $<0.001$ & $<0.001$ & $<0.001$ & $<0.001$ & $<0.01$ & 0.03 & 0.03 \\
\hline LRYGB & $<0.001$ & $<0.001$ & $<0.001$ & $<0.001$ & $<0.001$ & $<0.001$ & $<0.001$ & $<0.001$ & $<0.001$ & $<0.001$ & $<0.001$ \\
\hline LSG & 0.78 & $<0.001$ & $<0.001$ & $<0.001$ & $<0.001$ & $<0.01$ & - & - & - & - & - \\
\hline \multicolumn{12}{|c|}{ Number of patients remaining in study } \\
\hline LAGB & 4,208 & 4,208 & 4,208 & 3,681 & 3,141 & 2,649 & 2,139 & 1724 & 1366 & 1033 & 774 \\
\hline LRYGB & 4,308 & 4,308 & 4,308 & 3,683 & 3,053 & 2,479 & 1,938 & 1520 & 1158 & 905 & 684 \\
\hline LSG & 545 & 545 & 545 & 483 & 372 & 249 & 78 & 34 & 6 & . & . \\
\hline Control & 9,061 & 9,061 & 9,061 & 8,660 & 8,013 & 7,150 & 5,947 & 4,988 & 4,085 & 3,329 & 2,552 \\
\hline
\end{tabular}

LAGB, laparoscopic adjustable gastric band; LRYGB, laparoscopic Roux-en-Y gastric bypass; LSG, laparoscopic sleeve gastrectomy; LSM, least-squares means; y, year

* Medication costs are limited to the medications specifically used for the treatment of T2DM, dyslipidemia, and hypertension; medical costs include costs for all medical claims; combined treatment costs are equal to the sum of the medication costs and medical costs

**Result is adjusted least-squares means prediction from a Mixed Model for Repeated Measures; the models adjusted for baseline Charlson Comorbidity Index and counts of medications for type 2 diabetes, hypertension, and dyslipidemia

dyslipidemia, and hypertension compared with the overall study cohorts

\section{Healthcare Costs among Patients with T2DM}

The results of the adjusted analyses of healthcare costs among patients with T2DM are shown in Table 4.In the LRYGB cohort, adjusted monthly combined treatment costs decreased from $\$ 1,297$ during the baseline period to a low of $\$ 1,018$ at 4 years of follow-up. The adjusted monthly costs for T2DM medications were higher among the LAGB and LRYGB cohorts than the control cohort during the baseline period, but were significantly lower than that of the control cohort during follow-up. The lrygbcohort had the greatest reductions in adjusted monthly costs for T2DM medications.

\section{Healthcare Costs among Patients with T2DM Using Insulin}

The results of the adjusted analyses of healthcare costs among patients with T2DM using insulin are shown in Table 5. In the LRYGB cohort, adjusted monthly combined treatment costs decreased from $\$ 1,945$ during the baseline period to $\$ 1,446$ at three years of follow-up. As observed in the other analyses, adjusted monthly costs for medications for T2DM, dyslipidemia, and hypertension were higher in the LAGB and lrygbcohorts than the control cohort during the baseline period, but were significantly lower than that of the control cohort during followup. The lrygbcohort had the greatest reductions in adjusted monthly medication costs. 
Table 3: Adjusted analyses of healthcare costs (constant 2013 USD) among patients with type 2 diabetes, dyslipidemia, and hypertension Half-yearly panel period after end of 60-day post-surgery recovery period

\begin{tabular}{|c|c|c|c|c|c|c|c|c|c|c|c|}
\hline Cohort & $\begin{array}{c}\text { Index } \\
\text { minus } 180 \\
\text { days }\end{array}$ & $0-0.5 y$ & $>0.5 y-1 y$ & $>1 y-1.5 y$ & $>1.5-2 y$ & $>2 y-2.5 y$ & $>2.5 y-3 y$ & $>3 y-3.5 y$ & $>3.5 y-4 y$ & $>4 y-4.5 y$ & $>4.5 y-5 y$ \\
\hline \multicolumn{12}{|c|}{ Adjusted Monthly Combined Treatment Costs*, LSM** } \\
\hline LAGB & $\$ 1,433$ & $\$ 1,261$ & $\$ 1,409$ & $\$ 1,483$ & $\$ 1,280$ & $\$ 1,687$ & $\$ 1,706$ & $\$ 2,023$ & $\$ 1,813$ & $\$ 1,637$ & $\$ 1,597$ \\
\hline LRYGB & $\$ 1,517$ & $\$ 1,048$ & $\$ 1,060$ & $\$ 1,077$ & $\$ 1,196$ & $\$ 1,111$ & $\$ 1,189$ & $\$ 1,141$ & $\$ 1,183$ & $\$ 1,448$ & $\$ 1,527$ \\
\hline LSG & $\$ 1,427$ & $\$ 1,025$ & $\$ 1,072$ & $\$ 1,063$ & $\$ 1,455$ & $\$ 1,188$ & - & - & - & - & - \\
\hline Control & $\$ 1,480$ & $\$ 1,634$ & $\$ 1,601$ & $\$ 1,670$ & $\$ 1,702$ & $\$ 1,716$ & $\$ 1,727$ & $\$ 1,854$ & $\$ 1,876$ & $\$ 2,099$ & $\$ 2,315$ \\
\hline \multicolumn{12}{|c|}{ P-values vs. control cohort } \\
\hline LAGB & 0.44 & $<0.01$ & 0.16 & 0.19 & $<0.01$ & 0.86 & 0.91 & 0.37 & 0.76 & 0.05 & $<0.01$ \\
\hline LRYGB & 0.51 & $<0.001$ & $<0.001$ & $<0.001$ & $<0.001$ & $<0.001$ & $<0.01$ & $<0.001$ & 0.001 & $<0.01$ & $<0.01$ \\
\hline LSG & 0.73 & 0.07 & 0.11 & 0.09 & 0.54 & 0.26 & - & - & - & - & - \\
\hline \multicolumn{12}{|c|}{ Adjusted Monthly Medical Costs*, LSM** } \\
\hline LAGB & $\$ 871$ & 889 & $\$ 1,040$ & 1,128 & $\$ 939$ & $\$ 1,341$ & $\$ 1,357$ & $\$ 1,663$ & $\$ 1,451$ & 1,282 & $\$ 1,278$ \\
\hline LRYGB & $\$ 916$ & $\$ 811$ & $\$ 855$ & $\$ 890$ & $\$ 1,001$ & $\$ 920$ & $\$ 990$ & $\$ 942$ & $\$ 955$ & $\$ 1,229$ & $\$ 1,309$ \\
\hline LSG & $\$ 919$ & 753 & $\$ 818$ & 782 & $\$ 1,197$ & $\$ 888$ & - & - & - & - & - \\
\hline Control & $\$ 1,037$ & $\$ 1,126$ & $\$ 1,102$ & $\$ 1,172$ & $\$ 1,204$ & $\$ 1,215$ & $\$ 1,232$ & $\$ 1,357$ & $\$ 1,383$ & 1,602 & $\$ 1,792$ \\
\hline \multicolumn{12}{|c|}{ P-values vs. control cohort } \\
\hline LAGB & $<0.01$ & 0.07 & 0.64 & 0.75 & 0.07 & 0.42 & 0.47 & 0.1 & 0.74 & 0.17 & 0.05 \\
\hline LRYGB & 0.01 & $<0.01$ & 0.04 & 0.03 & 0.14 & 0.05 & 0.14 & 0.02 & 0.04 & 0.11 & 0.06 \\
\hline LSG & 0.37 & 0.25 & 0.38 & 0.26 & 0.99 & 0.47 & - & - & - & - & - \\
\hline \multicolumn{12}{|c|}{ Adjusted Monthly Medication Costs*, LSM** } \\
\hline LAGB & $\$ 562$ & 372 & $\$ 369$ & 352 & $\$ 334$ & $\$ 336$ & $\$ 341$ & $\$ 352$ & $\$ 354$ & 344 & $\$ 310$ \\
\hline LRYGB & $\$ 602$ & 238 & $\$ 206$ & 191 & $\$ 196$ & $\$ 188$ & $\$ 195$ & $\$ 191$ & $\$ 218$ & 208 & $\$ 210$ \\
\hline LSG & $\$ 508$ & 271 & $\$ 255$ & 279 & $\$ 265$ & $\$ 294$ & - & - & - & - & - \\
\hline Control & $\$ 443$ & 507 & $\$ 499$ & 498 & $\$ 500$ & $\$ 505$ & $\$ 494$ & $\$ 492$ & $\$ 485$ & 487 & $\$ 511$ \\
\hline \multicolumn{12}{|c|}{ P-values vs. control cohort } \\
\hline LAGB & $<0.001$ & $<0.001$ & $<0.001$ & $<0.001$ & $<0.001$ & $<0.001$ & $<0.001$ & $<0.001$ & $<0.001$ & $<0.001$ & $<0.001$ \\
\hline LRYGB & $<0.001$ & $<0.001$ & $<0.001$ & $<0.001$ & $<0.001$ & $<0.001$ & $<0.001$ & $<0.001$ & $<0.001$ & $<0.001$ & $<0.001$ \\
\hline LSG & 0.28 & $<0.001$ & $<0.001$ & $<0.001$ & $<0.001$ & $<0.01$ & - & - & - & - & - \\
\hline \multicolumn{12}{|c|}{ Number of patients remaining in study } \\
\hline LAGB & 850 & 850 & 850 & 744 & 626 & 536 & 434 & 357 & 288 & 205 & 156 \\
\hline LRYGB & 1,117 & 1,117 & 1,117 & 948 & 782 & 633 & 485 & 373 & 275 & 211 & 164 \\
\hline LSG & 105 & 105 & 105 & 91 & 67 & 48 & 17 & 8 & 2 &. &. \\
\hline Control & 2,072 & 2,072 & 2,072 & 1,999 & 1,864 & 1,693 & 1,433 & 1,217 & 990 & 831 & 636 \\
\hline
\end{tabular}

LAGB, laparoscopic adjustable gastric band; LRYGB, laparoscopic Roux-en-Y gastric bypass; LSG, laparoscopic sleeve gastrectomy; LSM, least-squares means; y, year

* Medication costs are limited to the medications specifically used for the treatment of T2DM, dyslipidemia, and hypertension; medical costs include costs for all medical claims; combined treatment costs are equal to the sum of the medication costs and medical costs

**Result is adjusted least-squares means prediction from a Mixed Model for Repeated Measures; the models adjusted for baseline Charlson Co morbidity Index and counts of medications for type 2 diabetes, hypertension, and dyslipidemia

\section{Discussion}

In this large scale analysis patients who had laparoscopic bariatric surgery had sustained lower costs for medications for the treatment of T2DM, dyslipidemia, and hypertension compared to medically-managed controls. Of the 3 types of laparoscopic bariatric surgeries, LRYGB was associated with the greatest reductions in medication costs and was the only surgery type also associated with sustained lower combined treatment costs in comparison to medically-managed controls up to 5 years post-surgery. Among patients in study cohorts with obesity related co morbidities of T2DM, dyslipidemia, and hypertension, the savings in medication costs were greater than among all patients. LSG results are similar to LRYGB, but with only 2.5 years of post-surgery data, the results are not conclusive.

Similar to our study, Weiner, et al., did observe a sustained reduction in pharmacy costs [not specific for treatment of obesity related co morbidities] among bariatric surgery patients versus non-surgery controls up to 6 years post surgery. ${ }^{12}$ Two other observational studies of patients who underwent bariatric surgery have reported reductions in medication costs that are sustained relative to matched controls; $[13,14]$ in The Swedish Obese Subjects Study medication costs remained lower up to 20 
Assessing Real World Effect of Laparoscopic Bariatric Surgery on Healthcare Costs- a Retrospective Matched Cohort Study using a U.S. Administrative Claims Database

Table 4: Adjusted analyses of healthcare costs (constant 2013 USD) among patients with type 2 diabetes Half-yearly panel period after end of 60-day post-surgery recovery period

\begin{tabular}{|c|c|c|c|c|c|c|c|c|c|c|c|}
\hline Cohort & $\begin{array}{c}\text { Index minus } \\
180 \text { days }\end{array}$ & $0-0.5 y$ & $>0.5 y-1 y$ & $>1 y-1.5 y$ & $>1.5-2 y$ & $>2 y-2.5 y$ & $>2.5 y-3 y$ & $>3 y-3.5 y$ & $>3.5 y-4 y$ & $>4 y-4.5 y$ & $>4.5 y-5 y$ \\
\hline \multicolumn{12}{|c|}{ Adjusted Monthly Combined Treatment Costs*, LSM** } \\
\hline LAGB & $\$ 1,139$ & $\$ 1,209$ & $\$ 1,333$ & $\$ 1,209$ & $\$ 1,400$ & $\$ 1,410$ & $\$ 1,633$ & $\$ 1,587$ & $\$ 1,664$ & $\$ 1,516$ & $\$ 1,139$ \\
\hline LRYGB & $\$ 982$ & $\$ 1,027$ & $\$ 977$ & $\$ 1,092$ & $\$ 987$ & $\$ 1,058$ & $\$ 1,018$ & $\$ 1,123$ & $\$ 1,376$ & $\$ 1,298$ & $\$ 982$ \\
\hline LSG & $\$ 869$ & $\$ 996$ & $\$ 1,013$ & $\$ 1,457$ & $\$ 1,058$ & & & & & & $\$ 869$ \\
\hline Control & $\$ 1,425$ & $\$ 1,362$ & $\$ 1,472$ & $\$ 1,496$ & $\$ 1,484$ & $\$ 1,515$ & $\$ 1,703$ & $\$ 1,683$ & $\$ 1,821$ & $\$ 1,953$ & $\$ 1,425$ \\
\hline \multicolumn{12}{|c|}{ P-values vs. control cohort } \\
\hline LAGB & 0.2869 & 0.0059 & 0.1404 & 0.2028 & 0.013 & 0.4975 & 0.4393 & 0.6339 & 0.5528 & 0.3937 & 0.0363 \\
\hline LRYGB & 0.3069 & $<.0001$ & 0.0005 & $<.0001$ & 0.0002 & $<.0001$ & 0.0004 & $<.0001$ & 0.0006 & 0.0143 & 0.0014 \\
\hline LSG & 0.4471 & 0.0245 & 0.1389 & 0.0829 & 0.8972 & 0.2396 & & & & . & . \\
\hline \multicolumn{12}{|c|}{ Adjusted Monthly Medical Costs*, LSM** } \\
\hline LAGB & $\$ 756$ & 835 & $\$ 910$ & 1,033 & $\$ 921$ & $\$ 1,117$ & $\$ 1,127$ & $\$ 1,338$ & $\$ 1,276$ & 1,359 & $\$ 1,237$ \\
\hline LRYGB & $\$ 804$ & $\$ 794$ & $\$ 863$ & $\$ 820$ & $\$ 929$ & $\$ 818$ & $\$ 877$ & $\$ 827$ & $\$ 901$ & $\$ 1,171$ & $\$ 1,105$ \\
\hline LSG & $\$ 778$ & 668 & $\$ 801$ & 799 & $\$ 1,232$ & $\$ 807$ & & & & & \\
\hline Control & $\$ 900$ & $\$ 1,012$ & $\$ 954$ & $\$ 1,062$ & $\$ 1,083$ & $\$ 1,070$ & $\$ 1,101$ & $\$ 1,279$ & $\$ 1,250$ & 1,396 & $\$ 1,513$ \\
\hline \multicolumn{12}{|c|}{ P-values vs. control cohort } \\
\hline LAGB & 0.0002 & 0.0765 & 0.6591 & 0.7842 & 0.1495 & 0.6933 & 0.8404 & 0.6781 & 0.8713 & 0.8403 & 0.1786 \\
\hline LRYGB & 0.0072 & 0.0193 & 0.3245 & 0.0139 & 0.1444 & 0.0273 & 0.0769 & 0.0013 & 0.0284 & 0.2076 & 0.0428 \\
\hline LSG & 0.1873 & 0.1494 & 0.5221 & 0.3037 & 0.6139 & 0.4565 & & & & . & . \\
\hline \multicolumn{12}{|c|}{ Adjusted Monthly Medication Costs*, LSM** } \\
\hline LAGB & $\$ 451$ & 304 & $\$ 299$ & 297 & $\$ 281$ & $\$ 274$ & $\$ 274$ & $\$ 285$ & $\$ 304$ & 295 & $\$ 270$ \\
\hline LRYGB & $\$ 493$ & 188 & $\$ 164$ & 159 & $\$ 163$ & $\$ 164$ & $\$ 175$ & $\$ 180$ & $\$ 209$ & 191 & $\$ 182$ \\
\hline LSG & $\$ 395$ & 201 & $\$ 195$ & 211 & $\$ 227$ & $\$ 246$ & & & & & \\
\hline Control & $\$ 355$ & 412 & $\$ 408$ & 410 & $\$ 414$ & $\$ 414$ & $\$ 410$ & $\$ 417$ & $\$ 422$ & 412 & $\$ 424$ \\
\hline \multicolumn{12}{|c|}{ P-values vs. control cohort } \\
\hline LAGB & $<.0001$ & $<.0001$ & $<.0001$ & $<.0001$ & $<.0001$ & $<.0001$ & $<.0001$ & $<.0001$ & $<.0001$ & $<.0001$ & $<.0001$ \\
\hline LRYGB & $<.0001$ & $<.0001$ & $<.0001$ & $<.0001$ & $<.0001$ & $<.0001$ & $<.0001$ & $<.0001$ & $<.0001$ & $<.0001$ & $<.0001$ \\
\hline LSG & 0.3419 & $<.0001$ & $<.0001$ & $<.0001$ & 0.0002 & 0.0026 & & & & . & . \\
\hline \multicolumn{12}{|c|}{ Number of patients remaining in study } \\
\hline LAGB & 1,321 & 1,321 & 1,321 & 1,159 & 982 & 842 & 676 & 550 & 438 & 321 & 245 \\
\hline LRYGB & 1,664 & 1,664 & 1,664 & 1,419 & 1,179 & 953 & 738 & 575 & 428 & 331 & 255 \\
\hline LSG & 172 & 172 & 172 & 147 & 105 & 71 & 22 & 10 & 2 & . & . \\
\hline Control & 3,157 & 3,157 & 3,157 & 3,003 & 2,772 & 2,490 & 2,081 & 1,734 & 1,397 & 1,161 & 877 \\
\hline
\end{tabular}

LAGB, laparoscopic adjustable gastric band; LRYGB, laparoscopic Roux-en-Y gastric bypass; LSG, laparoscopic sleeve gastrectomy; LSM, least-squares means; y, year

*Medication costs are limited to the medications specifically used for the treatment of T2DM, dyslipidemia, and hypertension; medical costs include costs for all medical claims; combined treatment costs are equal to the sum of the medication costs and medical costs

**Result is adjusted least-squares means prediction from a Mixed Model for Repeated Measures; the models adjusted for baseline Charlson Comorbidity Index and counts of medications for type 2 diabetes, hypertension, and dyslipidemia

years following bariatric surgery.[14]Bariatric surgery appears to have a much greater impact on medication costs than other interventions, with a recent clinical trial reporting no statistically significant reductions in medication costs between participants ( $\mathrm{n}=79$, average BMI: $39.5 \mathrm{~kg} / \mathrm{m}^{2}$ ) with at least one obesity related co morbidity who received intensive behavioral and dietary treatment for 18 months in comparison to those who only received it for 6 months.[15]

A meta-analysis of several observational studies published after 2003 reported rates of T2DM remission ranging between $79 \%$ and $92 \%$ among patients who underwent bariatric surgery.[16] Additionally, rates of remission of dyslipidemia and hypertension ranged from $58 \%$ to $77 \%$, and $67 \%$ to $81 \%$, respectively.[16] For most studies included in the metaanalysis, remission rates were reported for the short term.[16] A nationally representative study estimated that medical spending among Medicare, Medicaid, and private insurance payers in 2006 was $42 \%$ higher for an obese vs. A normal weight person, of which a primary driver was obesity related medication costs.[17] Moreover, costs of treatment of obesity related co morbidities continues to rise in the U.S. The American Diabetes Association estimated the total economic cost of diagnosed diabetes in 2012 at $\$ 245$ billion, a $41 \%$ increase from the previous estimate of $\$ 174$ billion (in 2007 dollars).[18]Furthermore, an array of drugs are now available to treat not only inadequate glycemic 
Assessing Real World Effect of Laparoscopic Bariatric Surgery on Healthcare Costs-a Retrospective Matched Cohort Study using a U.S. Administrative Claims Database

Table 5: Adjusted analyses of healthcare costs (constant 2013 USD) among patients with type 2 diabetes using insulin Half-yearly panel period after end of 60-day post-surgery recovery period

\begin{tabular}{|c|c|c|c|c|c|c|c|c|c|c|c|}
\hline Cohort & $\begin{array}{c}\text { Index } \\
\text { minus } 180 \\
\text { days }\end{array}$ & $0-0.5 y$ & $>0.5 y-1 y$ & $>1 y-1.5 y$ & $>1.5-2 y$ & $>2 y-2.5 y$ & $>2.5 y-3 y$ & $>3 y-3.5 y$ & $>3.5 y-4 y$ & $>4 y-4.5 y$ & $>4.5 y-5 y$ \\
\hline \multicolumn{12}{|c|}{ Adjusted Monthly Combined Treatment Costs*, LSM** } \\
\hline LAGB & $\$ 1,884$ & $\$ 1,578$ & $\$ 1,573$ & $\$ 1,681$ & $\$ 1,796$ & $\$ 2,480$ & $\$ 2,861$ & $\$ 2,590$ & $\$ 2,432$ & $\$ 2,757$ & $\$ 2,580$ \\
\hline LRYGB & $\$ 1,945$ & $\$ 1,322$ & $\$ 1,385$ & $\$ 1,347$ & $\$ 1,538$ & $\$ 1,466$ & $\$ 1,446$ & $\$ 1,428$ & $\$ 1,611$ & $\$ 1,923$ & $\$ 2,114$ \\
\hline LSG & $\$ 1,870$ & $\$ 1,103$ & $\$ 1,194$ & $\$ 1,511$ & & & & & & & \\
\hline Control & $\$ 1,776$ & $\$ 2,043$ & $\$ 1,907$ & $\$ 2,096$ & $\$ 2,153$ & $\$ 2,060$ & $\$ 2,243$ & $\$ 2,460$ & $\$ 2,562$ & $\$ 2,723$ & $\$ 2,915$ \\
\hline \multicolumn{12}{|c|}{ P-values vs. control cohort } \\
\hline LAGB & 0.3867 & 0.0922 & 0.2262 & 0.1491 & 0.2429 & 0.1963 & 0.0804 & 0.7375 & 0.7631 & 0.9464 & 0.56 \\
\hline LRYGB & 0.1155 & 0.0023 & 0.0274 & 0.0025 & 0.019 & 0.037 & 0.0127 & 0.0038 & 0.0204 & 0.0786 & 0.1213 \\
\hline LSG & 0.7508 & 0.1487 & 0.2733 & 0.381 & & & & & & . & . \\
\hline \multicolumn{12}{|c|}{ Adjusted Monthly Medical Costs*, LSM** } \\
\hline LAGB & $\$ 1,017$ & 990 & $\$ 1,007$ & 1,127 & $\$ 1,242$ & $\$ 1,944$ & $\$ 2,329$ & $\$ 2,058$ & $\$ 1,867$ & 2,230 & $\$ 2,127$ \\
\hline LRYGB & $\$ 1,031$ & $\$ 965$ & $\$ 1,075$ & $\$ 1,059$ & $\$ 1,244$ & $\$ 1,162$ & $\$ 1,118$ & $\$ 1,089$ & $\$ 1,221$ & $\$ 1,575$ & $\$ 1,772$ \\
\hline LSG & $\$ 1,118$ & 782 & $\$ 853$ & 1,194 & & & & & & & \\
\hline Control & $\$ 1,060$ & $\$ 1,289$ & $\$ 1,162$ & $\$ 1,382$ & $\$ 1,426$ & $\$ 1,337$ & $\$ 1,532$ & $\$ 1,699$ & $\$ 1,837$ & 2,023 & $\$ 2,214$ \\
\hline \multicolumn{12}{|c|}{ P-values vs. control cohort } \\
\hline LAGB & 0.6909 & 0.269 & 0.5653 & 0.3633 & 0.537 & 0.0564 & 0.0217 & 0.3458 & 0.944 & 0.6714 & 0.8784 \\
\hline LRYGB & 0.7566 & 0.1622 & 0.706 & 0.1806 & 0.4779 & 0.5315 & 0.1868 & 0.0818 & 0.1269 & 0.3177 & 0.3854 \\
\hline LSG & 0.8197 & 0.4259 & 0.6278 & 0.7728 & & & & & & . & . \\
\hline \multicolumn{12}{|c|}{ Adjusted Monthly Medication Costs*, LSM** } \\
\hline LAGB & $\$ 867$ & 588 & $\$ 566$ & 553 & $\$ 549$ & $\$ 532$ & $\$ 533$ & $\$ 533$ & $\$ 570$ & 527 & $\$ 456$ \\
\hline LRYGB & $\$ 913$ & 358 & $\$ 311$ & 291 & $\$ 296$ & $\$ 299$ & $\$ 318$ & $\$ 323$ & $\$ 376$ & 334 & $\$ 337$ \\
\hline LSG & $\$ 752$ & 321 & $\$ 341$ & 315 & & & & & & & \\
\hline Control & $\$ 716$ & 755 & $\$ 745$ & 717 & $\$ 729$ & $\$ 725$ & $\$ 708$ & $\$ 757$ & $\$ 718$ & 693 & $\$ 692$ \\
\hline \multicolumn{12}{|c|}{ P-values vs. control cohort } \\
\hline LAGB & 0.0016 & $<.0001$ & $<.0001$ & 0.0002 & $<.0001$ & $<.0001$ & 0.0003 & $<.0001$ & 0.0071 & 0.006 & 0.0005 \\
\hline LRYGB & $<.0001$ & $<.0001$ & $<.0001$ & $<.0001$ & $<.0001$ & $<.0001$ & $<.0001$ & $<.0001$ & $<.0001$ & $<.0001$ & $<.0001$ \\
\hline LSG & 0.7533 & $<.0001$ & $<.0001$ & $<.0001$ & & & & & . & . & . \\
\hline \multicolumn{12}{|c|}{ Number of patients remaining in study } \\
\hline LAGB & 277 & 277 & 277 & 247 & 211 & 182 & 153 & 126 & 97 & 69 & 50 \\
\hline LRYGB & 464 & 464 & 464 & 403 & 342 & 271 & 206 & 159 & 110 & 87 & 68 \\
\hline LSG & 35 & 35 & 35 & 33 & 27 & 17 & 7 & 3 & . & . & . \\
\hline Control & 538 & 538 & 538 & 515 & 467 & 414 & 326 & 259 & 215 & 174 & 126 \\
\hline
\end{tabular}

LAGB, laparoscopic adjustable gastric band; LRYGB, laparoscopic Roux-en-Y gastric bypass; LSG, laparoscopic sleeve gastrectomy; LSM, least-squares means; y, year

*Medication costs are limited to the medications specifically used for the treatment of T2DM, dyslipidemia, and hypertension; medical costs include costs for all medical claims; combined treatment costs are equal to the sum of the medication costs and medical costs

**Result is adjusted least-squares means prediction from a Mixed Model for Repeated Measures; the models adjusted for baseline Charlson Comorbidity Index and counts of medications for type 2 diabetes, hypertension, and dyslipidemia

control, but multiple complications of T2DM.Further study of the influence of bariatric surgery procedures on medication costs for obesity related co morbidities in the current and future healthcare landscape are warranted, along with additional longterm studies of the impact on cardiovascular events and other co morbidity related outcomes.

Given the potential cost savings that may be realized by laparoscopic bariatric surgery, the question arises as to when the economic Return On Investment (ROI) in such surgeries may yield an economic net benefit (i.e., when long-term savings exceed the initial costs of surgery). Two previous studies which addressed the ROI of laparoscopic bariatric surgery reported times to full ROI(economic net benefit) ranging from 1.25 to 5 years when comparing to morbidly obese patients who do not undergo bariatric surgery; with patients having baseline diabetes experiencing shorter durations of time to full ROI.[19,20] We conducted anroi analysis based on the present study findings in which we treated the costs incurred from 1 month before the bariatric surgery plus the cost incurred for the bariatric surgery as the initial investment, and treated the sum of cost differences between the surgical and control cohorts over time, applying a $3 \%$ annual discount rate, as the returns on the investment. We found that over the 5-year period, time to full ROI was realized for 
the LRYGB cohort in approximately 4 years among patients with T2DM and in approximately 3 years among patients with T2DM using insulin; this finding was robust to a more conservative discount rate of $5 \%$. Full ROI was not realized over the study period for the LAGB cohort in any of the groups of interest, or for the LRYGB cohort in the all patients group or in patients with T2DM, dyslipidemia, and hypertension. The LSG cohort was not subjected to the ROI analysis due to the limited sample size and follow-up duration.

\section{Limitations}

The results of this observational study must be presented with some limitations. First, the attrition rate of the study population was $34 \%$ after 3 years and $72 \%$ after 5 years. Loss to follow-up attributable to treatment failure may have caused an overestimation of differences in healthcare costs among patients who underwent laparoscopic bariatric surgery and medicallymanaged controls. Additionally, the follow-up period may not have been sufficient to examine the sustainability of differences in healthcare costs, especially for patients who had LSG.

Although LSG appeared to provide an economic benefit earlyon, due to few observations and short follow-up period it is not possible to draw meaningful results for this study population beyond 2.5 years. For this study, we attempted in multiple ways to control for differences in patient characteristics with advanced patient matching techniques; however some bias may still be present. The ICD-9-CM code, 278.00 is limited as it refers to "unspecified obesity" and does not refer to a specific BMI measurement. However, the majority [ 88\%] of all study cohorts had the ICD-9-CM code of 278.01, which refers to severe obesity with a BMI $>40 \mathrm{~kg} / \mathrm{m}^{2}$. Although it can be observed indirectly relative to controls, we did not directly compare differences in healthcare costs among patients who underwent LAGB, LRYGB, and LSG.ROI analyses are subject to uncertainty, and future analyses are needed to understand the ROI of LSG. Lastly, although the study population for this analysis was large and diverse, the results may not be generalizable to those older than 65 years or those with other types of insurance, such as Medicaid.

\section{Conclusions}

In this retrospective analysis of a large real world claims database, patients who had laparoscopic bariatric surgery incurred lower medication costs for the treatment of T2DM, dyslipidemia, and hypertension compared to medically-managed controls up to 5 years post-surgery. LRYGB was associated with the greatest reduction in medication costs and sustained lower combined treatment costs in comparison to medicallymanaged controls over the 5 year follow-up period. LAGB did not demonstrate sustained lower cost vs. control, and more study is needed to evaluate the long term impact of LSG.

\section{Acknowledgements}

We would like to acknowledge Jay Lin and Melissa LingohrSmith from Novosys Health in the editorial support and review of this manuscript, which was financially supported by Johnson
\& Johnson. We would also like to thank Stephen Johnston, an employee of Johnson \& Johnson, for assistance with review and revision of this manuscript.

\section{Disclosure}

This study and development of this manuscript was supported by Johnson \& Johnson. John Dawson and Eric Bour are consultants for Johnson \& Johnson. Andrew Yoo and Gang Li are employees of Johnson \& Johnson. Anuprita Patkar and Natalie Heidrich are employees of Ethicon, a Johnson \& Johnson company.

\section{References}

1. Ogden CL, Carroll MD, Kit BK, Flegal KM. Prevalence of obesity in the United States, 2009-2010. NCHS Data Brief. 2012;82:1-8.

2. Buchwald H, Consensus Conference Panel. Consensus Statement: Bariatric Surgery for Morbid Obesity: Health Implications for Patients, Health Professionals, and Third-party payers. SurgObesRelat Dis. 2005;1(3):371-381.

3. Cawley J, Meyerhoefer C. The medical care costs of obesity: an instrumental variables approach. J Health Econ. 2012;31(1):219230. doi: 10.1016/j.jhealeco.2011.10.003.

4. Apovian CM. The clinical and economic consequences of obesity. Am J Manag Care. 2013;19 (10 Suppl):s219-228.

5. Spieker EA, Pyzocha N. Economic impact of obesity. Prim Care. 2016;43(1):83-95. doi: 10.1016/j.pop.2015.08.013.

6. Mechanick JI, Youdim A, Jones DB; American Association of Clinical Endocrinologists; Obesity Society, American Society for Metabolic \& Bariatric Surgery. Clinical practice guidelines for the perioperative nutritional, metabolic, and nonsurgical support of the bariatric surgery patient-2013 update: cosponsored by American Association of Clinical Endocrinologists, the Obesity Society, and American Society for Metabolic \& Bariatric Surgery. EndocrPract. 2013;19(2):337-372. doi: 10.4158/EP12437.GL.

7. American Society for Metabolic \& Bariatric Surgery. Fact Sheet: Metabolic and Bariatric Surgery. May 2013.

8. Khan S, Rock K, Baskara A, Qu W, Nazzal M, Ortiz J. Trends in bariatric surgery from 2008 to 2012. Am J Surg. 2016;211(6):1041-1046. Doi: 10.1016/j.amjsurg.2015.10.012.

9. Colquitt JL, Pickett K, Loveman E, Frampton, GK. Surgery for weight loss in adults. Cochrane Database Sys Rev. 2014;8(8):CD003641. doi: 10.1002/14651858.CD003641.

10. Nguyen NT, Masoomi H, Magno CP, Nguyen XM, Laugenour K, Lane J. Trends in use of bariatric surgery, 2003-2008. J Am Coll Surg. 2011;213(2):261-266. doi: 10.1016/j.jamcollsurg.2011.04.030.

11. Hinojosa MW, Varela JE, Parikh D, Smith BR, Nguyen XM, Nguyen NT. National trends in use and outcome of laparoscopic adjustable gastric banding. SurgObesRelat Dis. 2009;5(2):150-155. doi: 10.1016/j. soard.2008.08.006.

12. Weiner JP, Goodwin SM, Chang HY, Bolen SD, Richards TM,Johns $\mathrm{RA}$, et al. Impact of bariatric surgery on health care costs of obese persons: a 6-year follow-up of surgical and comparison cohorts using health plan data. JAMA Surg. 2013;148(6):555-562. doi: 10.1001/ jamasurg.2013.1504.

13. Maciejewski ML, Livingston EH, Smith VA, Kahwati LC, Henderson WG, Arterburn DE. Health expenditures among high-risk patients after gastric bypass and matched controls. Arch Surg. 2012;147(7):633640. doi: 10.1001/archsurg.2012.818. 
14. Neovius M, Narbro K, Keating C, peltonen M, sjoholm K,Agren $\mathrm{G}$, et al. Health care use during 20 years following bariatric surgery. JAMA. 2012;308(11):1132-1141.

15. Tsai AG, Juarez-Columga E, Felton S, Speer RB, Bessesen DH, Atherly AJ. Medication costs during an 18 month clinical trial of obesity treatment among patients encountered in primary care. BMC Obes. 2015;2:24. doi: 10.1186/s40608-015-0054-4.

16. Chang SH, Stoll CR, Song J, Varela JE, Eagon CJ, Colditz GA. The effectiveness and risks of bariatric surgery: an updated systematic review and meta-analysis, 2003-2012. JAMA Surg. 2014;149(3):275287. doi: 10.1001/jamasurg.2013.3654.

17. Finkelstein EA, Trogdon JG, Cohen JW, Dietz W. Annual medical spending attributable to obesity: payer-and service-specific estimates. Health Aff (Millwood). 2009;28(5):822-831. Doi: 10.1377/ hlthaff.28.5.w822.

18. American Diabetes Association. Economic costs of diabetes in the U.S. in 2012. Diabetes Care. 2013;36(4):1033-1046. doi: 10.2337/dc122625.

19. Cremieux PY, Buchwald H, Shikora SA, Ghosh A, Yang HE, Buessing M. A study on the economic impact of bariatric surgery. Am J Manag Care. 2008;14(9):589-596.

20. Finkelstein EA, Allaire BT, Globe D, Dixon JB. The business case for bariatric surgery revisited: a non-randomized case-control study. PLoS One. 2013;8(9):e75498. doi: 10.1371/journal.pone.0075498. 
Assessing Real World Effect of Laparoscopic Bariatric Surgery on Healthcare Costs- a Retrospective Matched Cohort Study using a U.S. Administrative Claims Database

Supplemental Table 1: Explanation of study periods

\begin{tabular}{|l|l|c|}
\hline Study period/point & \multicolumn{1}{|c|}{ Time window/point } & \multicolumn{1}{c|}{ Measures } \\
\hline Index date & $\begin{array}{l}\text { Date of laparoscopic bariatric surgery for surgical cohort patients, matched } \\
\text { assigned date for control cohort patients }\end{array}$ & $\begin{array}{l}\text { Type of laparoscopic bariatric surgery for surgical } \\
\text { cohort patients }\end{array}$ \\
\hline $\begin{array}{l}\text { Baseline period } \\
\text { Post-surgery } \\
\text { recovery period }\end{array}$ & -360 days to Index date & Baseline demographics and clinical characteristics \\
\hline $\begin{array}{l}\text { Start of follow-up } \\
\text { period }\end{array}$ & Index date through + 60 days & None \\
\hline $\begin{array}{l}\text { First half-yearly } \\
\text { panel }\end{array}$ & Start of follow-up period through + 6 months & Healthcare costs \\
\hline $\begin{array}{l}\text { Second half-yearly } \\
\text { panel }\end{array}$ & 7 th month of follow-up period through end of first year of follow-up period & Healthcare costs \\
\hline $\begin{array}{l}\text { Third half-yearly } \\
\text { panel }\end{array}$ & Start of 2nd year of follow-up period through + 6 months & Healthcare costs \\
\hline \multicolumn{1}{|c|}{ Etc. } & Etc. & Healthcare costs \\
\hline
\end{tabular}

